\title{
Non-invasive brain stimulation: enhancing motor and cognitive functions in healthy old subjects
}

\section{Maximo Zimerman and Friedhelm C. Hummel*}

Brain Imaging and Neurostimulation Laboratory, Abteilung für Neurologie, Universitätsklinikum Hamburg-Eppendorf, Hamburg, Germany

\section{Edited by:}

Arthur F. Kramer, University of Illinois at

Urbana-Champaign, USA

\section{Reviewed by:}

Diane Beck, University of Illinois at

Urbana-Champaign, USA

Ruth Ann Atchley, University of Kansas, USA

\section{*Correspondence:}

Friedhelm C. Hummel, Brain Imaging and Neurostimulation Laboratory,

Department of Neurology, University Medical Center Hamburg-Eppendorf, 20246 Hamburg, Germany.

e-mail:f.hummel@uke.uni-hamburg.de
Healthy aging is accompanied by changes in cognitive and motor functions that result in impairment of activities of daily living. This process involves a number of modifications in the brain and is associated with metabolic, structural, and physiological changes; some of these serving as adaptive responses to the functional declines. Up to date there are no universally accepted strategies to ameliorate declining functions in this population. An essential basis to develop such strategies is a better understanding of neuroplastic changes during healthy aging. In this context, non-invasive brain stimulation techniques, such as transcranial direct current or transcranial magnetic stimulation, provide an attractive option to modulate cortical neuronal assemblies, even with subsequent changes in neuroplasticity. Thus, in the present review we discuss the use of these techniques as a tool to study underlying cortical mechanisms during healthy aging and as an interventional strategy to enhance declining functions and learning abilities in aged subjects.

Keywords: healthy aging, TMS, tDCS, brain plasticity, motor, cognition, brain stimulation

\section{INTRODUCTION}

During the last century, average life expectancy in developed countries was prolonged approximately 30 years. In addition, a significant decline in fertility generated a shift in the distribution of the population with important socio-economic, political, and public health consequences (UN-Report, 2005). The fact that there are more old people today than ever before, and that this tendency is expected to increase further, underlines the importance of understanding the mechanisms of healthy aging as well as developing novel innovative strategies to adapt for age-relate declines.

Besides age-associated diseases, like cardiovascular diseases or cancer (Balducci and Extermann, 2000), age-related neurobiological changes with consecutive declines in cognitive functions, perceptual, and motor abilities impair activities of daily living, independence, and quality of life (Logsdon et al., 2002; Craik and Bialystok, 2006). In cognition, age-related deficits encompass multiple domains, including attention, memory, reasoning, and executive functions (Celsis, 2000; Hogan et al., 2006). Age-related motor impairments are also ubiquitous, with deficits in the planning, the execution and the control of movement (Krampe et al., 2002; Sawaki et al., 2003). Physiologically, aging consists of a dynamic process in the brain, involving a number of modifications associated with metabolic, structural, and functional changes, part of them hypothesized as adaptive responses to the functional declines (Burke and Barnes, 2006).

In recent years, different forms of non-invasive brain stimulation techniques have been explored in patients and healthy volunteers offering the attractive option to modulate neuronal plasticity and to improve behavior and learning processes (Hummel and Cohen, 2006; Nitsche et al., 2008; Reis et al., 2009). In the present review, we will focus on studies using non-invasive brain stimulation techniques to evaluate cortical mechanisms during healthy aging, especially the one involved in preserving cognitive and motor func- tions. Furthermore, the novel field of applying these techniques to increase behavior, neuronal plasticity and learning, e.g., in the sensorimotor domain as a model system, will be presented. Rather than attempting to be comprehensive in terms of reviewed work, this article intends to provide a clearly structured framework of the application of these techniques to understand and support plastic changes in the aging population.

\section{NON-INVASIVE BRAIN STIMULATION}

Two main non-invasive brain stimulation methods to modulate cortical excitability have proved to be promising to induce long-lasting plastic changes in the sensori motor and cognitive systems.

\section{REPETITIVE TRANSCRANIAL MAGNETIC STIMULATION}

Repetitive transcranial magnetic stimulation is a painless and noninvasive technique to stimulate the brain. It consists of brief electrical currents delivered to the brain by an insulated wire coil placed on the skull. Currents generate a transient, high-intensity magnetic field with lines of flux running perpendicular to the coil. If the coil is held over the subjects head, it induces a secondary electrical current in the brain perpendicularly to the magnetic field that is capable of depolarizing neurons. All cortical areas beneath the skull can be potentially modulated, however most studies have focused on the motor cortex where an electromyographic response (motor evoked potential [MEP]) to single-pulse TMS can be measured providing the opportunity to read out changes in cortical activation (for details, please see Hummel and Cohen, 2005; Hallett, 2007). Depending on the frequency, duration, strength of the magnetic field and shape of the coil, rTMS can enhance or suppress activity in underlying cortical regions (Hummel and Cohen, 2005; Fregni and Pascual-Leone, 2007). In general, "high-frequency stimulation" refers to frequencies above $5 \mathrm{~Hz}$ and is considered to produce an excitatory effect, whereas "low frequency stimulation" refers to 
frequencies below $1 \mathrm{~Hz}$ causing inhibition of cortical excitability (Fregni and Pascual-Leone, 2007). The shape of the coil determines whether rTMS is rather focal (figure of eight coil) or rather nonfocal (round coil). The duration of the stimulation (in the range of up to $30 \mathrm{~min}$ ) is related to the duration of the effect induced by rTMS, which can persist even after the end of stimulation for several minutes or even up to an hour (for a review, please see Hallett, 2007). Recently, an interesting, novel mode of application of rTMS, called "theta-burst stimulation" (TBS), has been introduced capable of conditioning the human cortex for up to $90 \mathrm{~min}$ using short (less than $1 \mathrm{~min}$ ) asynchronous high frequency rTMS trains (theta-frequency, about $5 \mathrm{~Hz}$ ). Applying theta bursts continuously induces an inhibitory effect, while applying intermittent theta-bursts provides an excitatory effect (Huang et al., 2005).

Although rTMS is a safe procedure, it has the potential to cause seizures even in normal individuals. Contraindications to TMS are generally related to exposure to the magnetic field and therefore similar to those for magnetic resonance imaging (MRI). The magnetic pulse may damage electronic devices, and metal objects will be subject to mechanical forces. Exclusion criteria before proceeding need to be carefully considered and include: implanted metal devices such as cardiac pacemakers or defibrillators, drug delivery pumps, acoustic devices such as cochlear implants or spinal cord and vagus nerve stimulators (Rossi et al., 2009). Furthermore, safety guidelines describing limits for combinations of frequency, intensity, and train length have been developed to avoid relevant side effects (Wassermann, 1998; Rossi et al., 2009).

\section{TRANSCRANIAL DIRECT CURRENT STIMULATION}

The transcranial application of weak direct current is an effective and relative simple method to polarize brain regions. It consists of a battery and a current regulator, attached by wires to the scalp with two pad electrodes. Transcranial direct current stimulation (tDCS) has been demonstrated to modulate human cerebral cortical functions inducing focal, prolonged, but reversible shifts of excitability (Nitsche et al., 2003b). Previous studies in animals have used intracortical or epidural electrodes to stimulate the brain. In the past few decades, however, transcranial application of low amplitude direct currents demonstrated to induce intracerebral current flow sufficiently large enough to be effective in altering cortical neuronal activity (Hummel and Cohen, 2005). The efficacy and duration of the tDCS-induced excitability changes in humans depend on length of the stimulation, size of the electrodes, and strength of the current (Hummel and Cohen, 2005; Nitsche et al., 2008), whereas, polarity is determined by the orientation of the electric field. For example, anode over the motor cortex and cathode over the contralateral supra-orbital region produces a lasting increase in motor cortical excitability when the current is applied for several minutes. In contrast, when the electrodes are reversed, a decrease in motor cortical excitability is seen (Nitsche et al., 2008).

A growing body of research demonstrated modulation of behavior after $\mathrm{tDCS}$ application in the motor, somatosensory, visual, and cognitive domains in healthy volunteers and neuropsychiatric disorders (Antal et al., 2004; Hummel et al., 2005, 2006; Boggio et al., 2010). Although tDCS is a painless method, at the beginning of the stimulation it can induce short-lasting tingling, burning sensations which fade away in seconds (Nitsche et al., 2008). This effect has been used to develop "Sham/placebo" stimulation protocols. Within these protocols tDCS will be started in both conditions, but faded out after $30 \mathrm{~s}$ in the sham condition, the time frame in which the above mentioned sensations also fade away. Thus, with this procedure subjects cannot distinguish between real and sham tDCS with the opportunity to blind both investigator and subjects in these experiments (Hummel and Cohen, 2005; Gandiga et al., 2006; Nitsche et al., 2008).

Both of the presented NIBS techniques can effectively modulate brain functions and both are painless and non-invasive. However, their underlying mechanisms differ (for a detailed description of the mechanism of action please see Fregni and Pascual-Leone, 2007; Hallett, 2007; Nitsche et al., 2008). Furthermore, TMS has superior spatial and temporal resolution. On the other side, with tDCS it is easier to perform double-blind, sham-controlled studies and to simultaneously apply behavioral tasks (Hummel and Cohen, 2005; Nitsche et al., 2008). For a comparison between both NIBS techniques, please see Table 1. Importantly, both, rTMS and tDCS, can induce long-term after-effects (up to $1-2 \mathrm{~h}$ after stimulation offset) on cortical excitability that may translate into physiological and behavioral modifications in combination with training (Hummel and Cohen, 2005). The intrinsic mechanisms underlying long-term effects of NIBS are not completely understood; however, it has been suggested to be analogous to long-term potentiation/depression (LTP/LTD) like effects demonstrated in the hippocampus after repeated activation of synaptic pathways (Hallett, 2007; Nitsche et al., 2008).

Modulation of the balance of neurotransmission can partially explain the neurophysiological effects of these techniques. NIBS may result in changes in inhibitory GABA-ergic as well as the excitatory glutamate-ergic intracortical systems which play a crucial role in the regulation of neuronal activity (Nitsche et al., 2003a). Recently, pharmacological studies described that D-cycloserine, a partial NMDA-agonist, selectively potentiates the duration of motor cortical excitability modulation by anodal tDCS (Nitsche et al., 2004). Furthermore, shortly after a 10-Hz rTMS application to the ipsilateral motor cortex, a focal increase of dopamine in the striatum was demonstrated in humans using PET (Strafella et al., 2003).

Additionally, another interesting concept has been put forward recently, namely that gene induction might be a potential mechanism by which NIBS may exert persistent plastic changes. Different studies support the view that rTMS modulates the expression of immediate early genes such as $c$-fos, $c$-jun, and astroglial gene expression in rodents (Fujiki and Steward, 1997; Hausmann et al., 2000). Another study exhibited that single sessions of rTMS increased $c$-fos mRNA expression predominately in the paraventricular nucleus of the thalamus and, in a lesser extent, in the frontal and cingulated cortices in animals (Ji et al., 1998). Using longer rTMS treatment protocols, a significant enhancement of Brain-derived neurotrophic factor (BDNF) mRNA in the hippocampus, parietal, and piriform cortices was described (Muller et al., 2000). Interestingly, recent research has demonstrated that BDNF might be a critical mediator of the magnitude of tDCSinduced effects. Fritsch et al. (2010) have shown that adult mice with deletion of the BDNF gene failed to exhibit the LTP-like effect in the motor cortex after tDCS exposure (Fritsch et al., 2010). As previously described, BDNF is a member of the neurotrophin family and plays a significant role in synaptogenesis and synaptic plastic mechanisms, which underlie learning and memory processes in the adult brain and 
in several pathologies (for a review, please see Binder and Scharfman, 2004). Until now, the most studied genetic influence consist of a single nucleotide polymorphism of the BDNF gene. A large number of the population has an amino acid substitution (valine to methionine) at codon 66 (val66met) and previous work correlated this genotype with both anatomical and behavioral consequences in healthy subjects (Egan et al., 2003). Recently, however, a reduction of cortical excitability changes after motor learning and decreased susceptibility to NIBS protocols was demonstrated in the val66met carriers (Kleim et al., 2006; Cheeran et al., 2008). These findings support the notion that BDNF is involved, at least in young subjects, in the induction of cortical plasticity changes by NIBS.

\section{MORPHOLOGICAL CHANGES OF THE AGING BRAIN}

During healthy aging, the brain experiences complex structural and biochemical changes, including modification in dendritic morphology, synaptic connectivity (Anderson and Rutledge, 1996), $\mathrm{Ca}^{2+}$ dysregulation (Toescu et al., 2004), gene expression (for review see Burke and Barnes, 2006) and a decrease in the availability and level of neurotransmitters (Roth and Joseph, 1994). Cholinergic and dopaminergic reductions are particularly pronounced compromising motor, attention, and memory processes (Volkow et al., 1998; Braver and Barch, 2002). Furthermore, extensive studies reported that the function of the dopaminergic system gradually declines as we grow older due to degeneration of dopaminergic neurons and receptors (Zaman et al., 2008).

Age-related decreases in white matter integrity appear to be a common process in the brain (Resnick et al., 2003; Stadlbauer et al., 2008). Recent morphological studies using diffusion tensor imaging (DTI, for review see Pierpaoli et al., 1996) in old healthy subjects have consistently shown a correlation between aging and reduction of fractional anisotropy, suggesting a rarefaction of directionally oriented axonal membranes, and increased mean diffusivity reflecting an alteration in cellular membranes and other structures hindering diffusion (Sullivan and Pfefferbaum, 2006). These age-related differences in white matter integrity are seen throughout the brain, with an increasing magnitude of the difference in anterior white matter structures compared to posterior regions, which most authors refer to as a "anterior-posterior gradient," with age-related changes occurring earlier in the frontal lobe (Bennett et al., 2010). The corpus callosum represents the largest white matter structure connecting the two hemispheres in the brain. Age-related changes in the topology of the corpus callosum primarily affect the genus; however, recent studies using more sensitive techniques of DTI also demonstrated changes in the splenium (Bastin et al., 2010). Until now, there is no consensus of the etiology and the functional repercussion of these changes in white matter structure, but recent data from healthy individuals and patients with mild cognitive impairments and dementia converge on highlighting correlations between cognitive performance and fractional anisotropy (Persson et al., 2006), indicating that the decline in white matter might be associated with cognitive impairment.

\section{AGE-RELATED CHANGES IN CORTICAL NEUROPHYSIOLOGY CORTICAL INHIBITION}

Changes in neurotransmission play a relevant role during healthy aging. Animal studies have consistently shown age-dependent changes in GABA-ergic cortical circuits (Yu et al., 2006; Schmidt et al., 2010). These findings are of great interest as GABA receptors are the most important inhibitory receptors in the central nervous system, playing a crucial role in the regulation of brain excitability and neuronal plasticity (Jones, 1993). In humans, GABA-ergic or glutamate-ergic neurotransmission can not be monitored directly. However, as demonstrated over the past two decades, TMS offers the interesting option to study these circuits in humans non-invasively. Intracortical inhibition (ICI, GABA-ergic) and intracortical facilitation (ICF, glutamate-ergic) can be evaluated in the motor cortex by so-called short-interval paired-pulse TMS protocols (see Kujirai et al., 1993; Chen et al., 2004). In brief, these widely used protocols involve a subthreshold conditioning stimulus followed by a suprathreshold test stimulus. The conditioning stimulus modulates the amplitude of the MEP produced by the test stimulus by mainly intracortical pathways. The test responses are inhibited at interstimulus intervals (ISIs) of 2-6 ms and they are facilitated at ISIs of 8-20 ms (for details please see Kujirai et al., 1993; Ziemann et al., 1996; Hanajima et al., 1998). These TMS protocols can not only be performed during rest but also while subjects perform a motor activity (Reynolds and Ashby, 1999; Hummel et al., 2009b; Heise et al., 2010).

To examine the effects of healthy aging on cortical excitability, several cross-sectional studies used these techniques to compare old with young healthy subjects. Overall, these studies yield inconsistent results and are difficult to compare one to another due to considerable differences regarding mean subject age, TMS technique used, small sample sizes, and great variability among the studies (Peinemann et al., 2001; Wassermann, 2002). Peinemann et al. (2001), demonstrated a reduction of ICI (indicating less inhibition) in a group of old healthy adults with respect to young controls, whereas ICF remained unchanged (Peinemann et al., 2001). Wasserman, however, found no correlation in ICI or ICF with age (Wassermann, 2002). More recently, an increase in ICI and a decrease of ICF has been reported under resting state and disappeared during a submaximal muscle contraction. It is important to mention that these measurements were performed in the non-dominant hand (McGinley et al., 2010).

Another physiological measure that correlates with intracortical inhibitory neurotransmission is the cortical silent period (CSP, for review please see Hallett, 1995). CSP refers to the interruption of voluntary muscle contraction by a single suprathreshold TMS pulse over the contralateral M1, evidence were provided that spinal inhibitory mechanisms contribute to the early part of the CSP (up to the first $50 \mathrm{~ms}$ ), but its later part is generated mainly by long-lasting inhibition originating within M1 (Chen et al., 1999). This type of inhibition, in contrast to ICI (GABA-A), has been suggested to be mediated by GABA-B receptors (Werhahn et al., 1999). Until now, three different studies demonstrated a consistent reduction in CSP in older adults, providing further evidence for an age-related decline in GABA-ergic inhibitory neurotransmission in the human motor cortex (Eisen et al., 1996; Sale and Semmler, 2005; Oliviero et al., 2006).

The presented studies evaluated intracortical excitability during rest and during submaximal contraction. However, given that old subjects often show impaired performance while executing a motor activity (Reynolds and Ashby, 1999; Hummel et al., 2009b; Heise et al., 2010) it would be valuable to evaluate these measures while 
subjects perform a motor task. To illustrate, employing the doublepulse paradigm during a simple finger movement revealed differences in the movement-related patterns of intracortical inhibition of healthy old subjects compared to young controls; with the old subjects showing disinhibition and a loss of modulation of cortical excitability. Taken together, changes in intracortical excitability indicate the presence of age-related modifications in GABA-ergic cortical systems. Whether these alterations of inhibition represent a compensatory mechanism to promote neuroplastic changes or a mere decrease in the effectivity of the system without any functional meaning is still an open question that has to be addressed in detail in upcoming studies.

Additionally, TMS provides not only the possibility to evaluate cortical excitability in the primary motor cortex but also interactions between different cortical areas, for example between both motor cortices. In healthy young subjects, there are balanced interhemispheric inhibitory interactions (IHI), which can be assessed by TMS paradigm over both motor cortex (Ferbert et al., 1992). Using two magnetic stimulating coils (paired-pulse measure), it is possible to study the effects of a conditioning stimulus applied to the M1 of one hemisphere on the MEP elicited by a test stimulus to the contralateral M1, inhibition occurs at intervals of 8-50 ms (Ferbert et al., 1992). Based on neurophysiological determinations and the observation that IHI is normal in stroke patients with a subcortical ischemic lesion of the corticospinal tract below the centrum semiovale (Boroojerdi et al., 1996), it was proposed that the inhibition was produced at a cortical level and mediated by transcallosal motor fibers. When a motor task is performed with one hand, the inhibitory influence targeting the ipsilateral hemisphere tends to increase (Duque et al., 2005). Talelli et al. (2008b) implemented this protocol at rest and during a low-strength isometric contraction in young and old healthy adults. The main findings were that during muscle activation the modulation of IHI was a function of age, suggesting that younger individuals showed stronger IHI, whereas in older individuals this phenomenon was attenuated and even reversed (Talelli et al., 2008b). Furthermore in another study combining fMRI and TMS, the same group probed that activity in the ipsilateral motor cortex during a hand motor task is greater when IHI from the contralateral hemisphere does not increase in relation to the task (Talelli et al., 2008a). These works provide further evidence that age-related changes in inhibitory cortical networks observed within M1 are associated with alterations in task-related brain activity in distributed and interconnected brain regions contributing in part to explain the finding of reduced lateralization during behavioral tasks in older individuals (Cabeza, 2002).

\section{NEURONAL PLASTICITY}

Neuronal plasticity is defined as any enduring change in cortical properties caused by environmental changes, lesions, or functional adaptation to new circumstances. Modification in human cortical plasticity has been well documented in physiological conditions such as training of new motor skills (Rosenkranz et al., 2007), manipulation of sensory input (Kaelin-Lang et al., 2002), as well as during pathological processes like response to amputation and stroke (Cohen et al., 1991; Hummel et al., 2005). In an attempt to understand if the basic mechanisms of neural plasticity are involved in maintaining motor functions of older people at a similar level to that of younger ones, few researchers started to transfer some of the "proof of principle" studies performed so far in young participants to the aged population. In this context, NIBS techniques were used as an effective method to modulate synaptic efficacy in human motor cortex to improve learning and behavior.

As recently suggested, the aged brain might react different to NIBS protocols compared to the young brain. For example, Todd et al. (2010) showed a reduced response to an inhibitory subthreshold rTMS protocol in healthy old subjects compared to young controls (Todd et al., 2010). In addition, the response to paired associative stimulation (PAS), a widely used protocol to induce LTP/LTD-like changes in the motor cortex by coupling somatosensory stimulation with motor cortex stimulation (Stefan et al., 2000), has been suggested to be dissimilar compared to young controls (Tecchio et al., 2008; Fathi et al., 2009; Pellicciari et al., 2009). Recent studies, however, have shown ambiguous results using this protocol in the aged populations: Tecchio et al. (2008) found an age-dependent decrease in motor cortex plasticity only in females after menopause, but not in age-matched male subjects (Tecchio et al., 2008). Pellicciari et al. (2009) reported that cortical excitability induced by PAS in somatosensory cortex increased in healthy aged population due to a possible compensatory mechanism in specific neuronal circuits (Pellicciari et al., 2009). In contrast other studies proposed a substantially decreased magnitude of PAS-induced motor cortical plasticity in old subjects (Muller-Dahlhaus et al., 2008; Fathi et al., 2009). Furthermore, Sawaki et al. (2003) showed a reduction of LTP-like mechanisms involved in use-dependent motor memory formation as a function of age (Sawaki et al., 2003). For a detailed summary, please see Table 2.

Although the published data is not completely conclusive, there is evidence provided suggesting that the response to NIBS might be different between young and old adults. There are different hypothesis to explain these differences. (1) Old subjects' neuronal systems do not respond to NIBS like the ones of young subjects. (2) The parameters for interventions for old subjects are not similar to the ones showed to be effective in young subjects. Thus, modified NIBS protocols might have to be tested in this population. (3) The neuronal system is already fully activated to compensate for agerelated functional deficits, thus, it cannot be further modulated by NIBS interventions.

\section{COMPENSATORY CORTICAL RECRUITMENT IN THE AGED BRAIN?}

Novel brain imaging techniques support the view that the neural system is continuously remodeled throughout life and after injury by experience and learning in response to activity and behavior (Jenkins et al., 1990). By applying functional neuroimaging to understand the processes underlying aging, a general finding has been described: in older individuals, activations tend to be weaker and more diffuse. In particular, hemispheric lateralization tends to be reduced during a behavioral task. Cabeza et al. (1997) found that activation of the prefrontal cortex during an episodic recall test based on word pairs was right-lateralized in young individuals but bilaterally represented in older adults (Cabeza et al., 1997). On the basis of these findings, in 2002 the HAROLD (Hemispheric Activity Reduction in Older Adults) model of age-related reduction of lateralization was introduced (Cabeza, 2002). This model suggests that, during cognitive tasks, low performing older subjects 
Table 1 | Summary of the main features of NIBS techniques (table modified from Gandiga et al., 2006).

\begin{tabular}{|c|c|c|}
\hline & TMS & tDCS \\
\hline Excitatory & High frequency $>5 \mathrm{~Hz}$ & Anodal stimulation \\
\hline Inhibitory & Low frequency $\sim 1 \mathrm{~Hz}$ & Cathodal stimulation \\
\hline Focality of stimulation & More focal & Less focal \\
\hline Design of sham-controlled double-blind studies & More difficult & Less difficult \\
\hline \multirow[t]{2}{*}{ Adverse effects } & Rare, if applied within the safety guidelines. & Rare, if applied within the safety guidelines. \\
\hline & $\begin{array}{l}\text { Possible headache, sensory discomfort, } \\
\text { minimal risk of seizures }\end{array}$ & Sometimes headache, no seizures described yet \\
\hline Time resolution & Excellent: milliseconds & Poor \\
\hline Cost & Higher & Lower \\
\hline
\end{tabular}

Table 2 | Induction of neuro-plasticity in old healthy subjects.

\begin{tabular}{|c|c|c|c|c|c|c|}
\hline Technique & Study type & $n$ & Outcome & Comments & Main findings & References \\
\hline PAS & $\begin{array}{l}\text { Parallel design, age, } \\
\text { and gender distributed }\end{array}$ & $\begin{array}{l}50 \text { ( } 25 \text { young, } \\
25 \text { old) }\end{array}$ & MEP & $\begin{array}{l}\text { N20 latency was not } \\
\text { measured }\end{array}$ & $\begin{array}{l}\text { Reduction of PAS effect only } \\
\text { in old females }\end{array}$ & $\begin{array}{l}\text { Tecchio et al. } \\
\text { (2008) }\end{array}$ \\
\hline PAS & $\begin{array}{l}\text { Single design (age } \\
\text { range 22-71 years) }\end{array}$ & 27 & MEP & $\begin{array}{l}\text { ISI equaled to } \\
\text { individual N20-latency }\end{array}$ & $\begin{array}{l}\text { PAS effect decreased with } \\
\text { age }\end{array}$ & $\begin{array}{l}\text { Muller-Dahlhaus } \\
\text { et al. (2008) }\end{array}$ \\
\hline rTMS & $\begin{array}{l}\text { Randomized, double } \\
\text { blind, parallel design }\end{array}$ & $\begin{array}{l}30 \text { (15 young, } \\
15 \text { elderly) }\end{array}$ & MEP & Sham condition & $\begin{array}{l}\text { rTMS effect absent in old } \\
\text { subjects }\end{array}$ & Todd et al. (2010) \\
\hline tDCS & $\begin{array}{l}\text { Two parallel group with } \\
\text { a cross-over design }\end{array}$ & $\begin{array}{l}8 \text { (mean age } \\
57 \text { years old) }\end{array}$ & MEP, ICl & $\begin{array}{l}\text { The subjects were a } \\
\text { control group }\end{array}$ & $\begin{array}{l}\text { Polarity-specific shift in MEP } \\
\text { but not in } \mathrm{SICl} \text { in elderly }\end{array}$ & $\begin{array}{l}\text { Quartarone et al. } \\
\text { (2007) }\end{array}$ \\
\hline
\end{tabular}

PAS, paired associative stimulation; rTMS, repetitive transcranial magnetic stimulation; tDCS, transcranial direct current stimulation; UDP, use-dependent plasticity; MEP, motor evoked potential; SEP, somatosensory evoked potential; ICl, intracortical inhibition.

showed similar unilateral prefrontal activation as young adults, while high-performing older subjects engaged a bilateral activation of the prefrontal cortex, indicating that recruiting additional cortical resources is one potential compensatory effect due to age-related functional declines. This model was supported by data acquired in the area of episodic memory (Cabeza et al., 1997), semantic memory (Stebbins et al., 2002), working memory (Reuter-Lorenz et al., 2000), and inhibitory control (Nielson et al., 2002).

Recently, it has been probed that changes in hemispheric asymmetry in older adults are reflective of a general aging phenomenon rather than a function-specific occurrence. In the motor domain, Mattay et al. (2002) found different patterns of activation during a visually paced button-press motor task in old and young participants. These additional areas of activation were seen in the ipsilateral sensorimotor cortex, putamen, and contralateral cerebellum of the old subjects (Mattay et al., 2002). In addition, Ward et al. (2008) investigated whether motor system activity during a task involving increasingly forceful hand grips was influenced by age. Subjects were asked to perform a simple isometric hand grip task with parametric modulation of force output. There were no age-related changes in BOLD response in contralateral M1, but consistent modifications have been described in ipsilateral M1. Furthermore, task-related activity co-varied positively with force output in a number of brain regions in young but not in the old group, indicating a reduced ability of old subjects to modulate activity in the appropriate motor networks (Ward et al., 2008). These differences in the cortical representation of motor acts has also been documented with electroencephalography (Sailer et al., 2000) and near-infrared spectroscopy techniques (Herrmann et al., 2006).

Increased cortical activity in old subjects during a motor or cognitive task with a decrease in lateralization, initially led to the assumption that the additional activation can potentially "compensate" for 
reduced activity in the circuits involved in the task (Cabeza et al., 2002) and much of the subsequent work has continued developing this hypothesis (Ward and Frackowiak, 2003). Nevertheless, another possible explanation can be taken into account regarding this over-recruitment in old subjects. That is the possibility that this engagement represents an inefficient use of the brain resources, with non-selective recruitment rather than functionally relevant additional activation. This assumption can not be ruled out based on the aforementioned functional imaging studies. As a matter of principle, neuroimaging techniques like EEG or fMRI are based on the analysis of associations between concomitant modulation of behavioral and neurophysiological parameters such as electric fields or metabolic blood flow changes. They are not geared to test causality between local brain function and behavioral output. The latter is the domain of inactivation techniques like the Wada test or, non-invasively, temporary interference approaches with rTMS or tDCS (Fregni and Pascual-Leone, 2007; Hallett, 2007; Nitsche et al., 2008).

In the following section such an approach is described in more detail and illustrated in Figure 1. Within this project the hypothesis was tested whether the ipsilateral motor cortex is functionally relevant, as suggested in several imaging studies, for the acquisition of a motor skill performed with the ipsilateral hand. To this purpose, cathodal (inhibitory) tDCS was used to down regulate the ipsilateral motor cortex during the training of a finger sequence motor task in old and young healthy adults (Figure 1). As hypothesized the disturbance of the ipsilateral motor cortex was followed by a functional impairment of the motor task indicated by a significant reduction of correctly played sequences in comparison to a sham stimulation condition. Furthermore this effect was only apparent in the old group and not in the young control group. Notably, the older the subject, the more prominent the disrupting effect of tDCS (Zimerman et al., 2008). Taken together these results strongly support the hypothesis that the ipsilateral motor cortex is causally involved in the acquisition and implementation of complex unimanual finger movements in old subjects but not in young ones. Moreover, it further substantiates the concept that, even in an aged population, an adaptable and plastic motor cortical network is able to respond to age-related degenerative changes in brain structure and function in order to maintain performance levels.

\section{ENHANCEMENT OF DECLINING FUNCTION IN OLD ADULTS}

According to previous studies performed in healthy subjects and in neuropsychiatric patients (e.g., stroke, Parkinson, and depression), non-invasive transcranial stimulation techniques have the potential to modulate activity in the targeted cortical region or in a dysfunctional network, to enhance function and restore an adaptive equilibrium for best behavioral outcome, or to suppress maladaptive plastic conditions (for review please see Fregni and Pascual-Leone, 2007; Hallett, 2007; Nitsche et al., 2008). Few studies examined the possibility to induce behavioral changes in old subjects. Most of the data found in the literature was obtained with younger subjects or patients with neurological disorders (Hummel et al., 2005; Cotelli et al., 2008; Wu et al., 2008) some of them affecting predominantly the aged population (e.g., stroke, Parkinson, and Alzheimer).
In the motor domain, in a double blinded, sham-controlled, cross-over study, Hummel et al. (2009) demonstrated that the application of anodal tDCS over the contralateral motor cortex (increase of motor cortical excitability) improved a set of skilled hand motor functions, mimicking daily life activities, measured with the JebsenTaylor hand function test in older subjects (Hummel et al., 2009a). The stimulation effect was more prominent in the skillful control of distal hand function (e.g., turning over cards, grasping small objects, and lifting small objects with a spoon) than of gross proximal functions. This is of special importance, given that the quality of life of old adults depend on an excellent functioning of the hand (Scherder et al., 2008). The results of the mentioned study are illustrated in Figure 2. A comparable concept was tested by Floel et al. (2008) using a pharmacological intervention and the same primary outcome. They found that levodopa facilitates the execution of complex hand activities in the old group. In this context, it is of interest that some authors proposed that tDCS-induced effects are in part mediated by a modulation of the dopaminergic system (Nitsche et al., 2006).

In another cognitive domain, Boggio et al. (2010) evaluated the effect of tDCS in a decision-making task in old subjects. They found that tDCS applied over both prefrontal cortex (left and right) modified the balance of risky and safe responses in a similar behavioral way, but with different topographical representation compared to a previous study performed in young subjects, supporting an agerelated reduction of lateralization as suggested in the HAROLD model (Boggio et al., 2010). Changes related to normal aging do occur in patients with Alzheimer disease (AD) as well. For instance, NIBS might improve cognitive variables in these patients as suggested by Ferrucci et al. (2008). They found that anodal tDCS over temporoparietal regions enhances the recognition memory capacity in patients with probable $\mathrm{AD}$ (mean age 75 years) (Ferrucci et al., 2008). Furthermore, Cotelli et al. (2008) demonstrated that rTMS applied to the dorso-lateral prefrontal cortex improves naming performance in old subjects (Cotelli et al., 2008).

Most of the studies performed so far in healthy subjects and patients have examined the effects of NIBS on electrophysiological measurements (e.g., intracortical excitability) or on behavioral tasks (e.g., Jebsen Taylor Hand function test), mainly in a single session approach. Within these approaches, transient behavioral improvements in the range of 10-20\% could be achieved (Hummel and Cohen, 2005, 2006). However, especially if NIBS is applied to ameliorate functional deficits, it is important to achieve longer lasting improvements with a larger magnitude. Maximum behavioral gains are optimized when brain stimulation is coupled with training, suggesting that it may act as a learning enhancer (Reis et al., 2009). In this context, recent evidence demonstrated in animal studies that training-induced cortical plasticity might be based on processes analogous to LTP/LTD like changes (Rioult-Pedotti et al., 2000), during acquisition of a new motor skill reorganization processes in M1 result in the formation of new cortical networks (Komiyama et al., 2010). These processes are most likely based on unmasking of excitatory connections within the cortex allowing rapid changes in motor representations (Huntley, 1997) by reducing the activity of existing inhibitory ones (Buonomano and Merzenich, 1998). According to this line, a recent elegant study demonstrated in mouse motor cortex slices that combining anodal 


\section{Design}
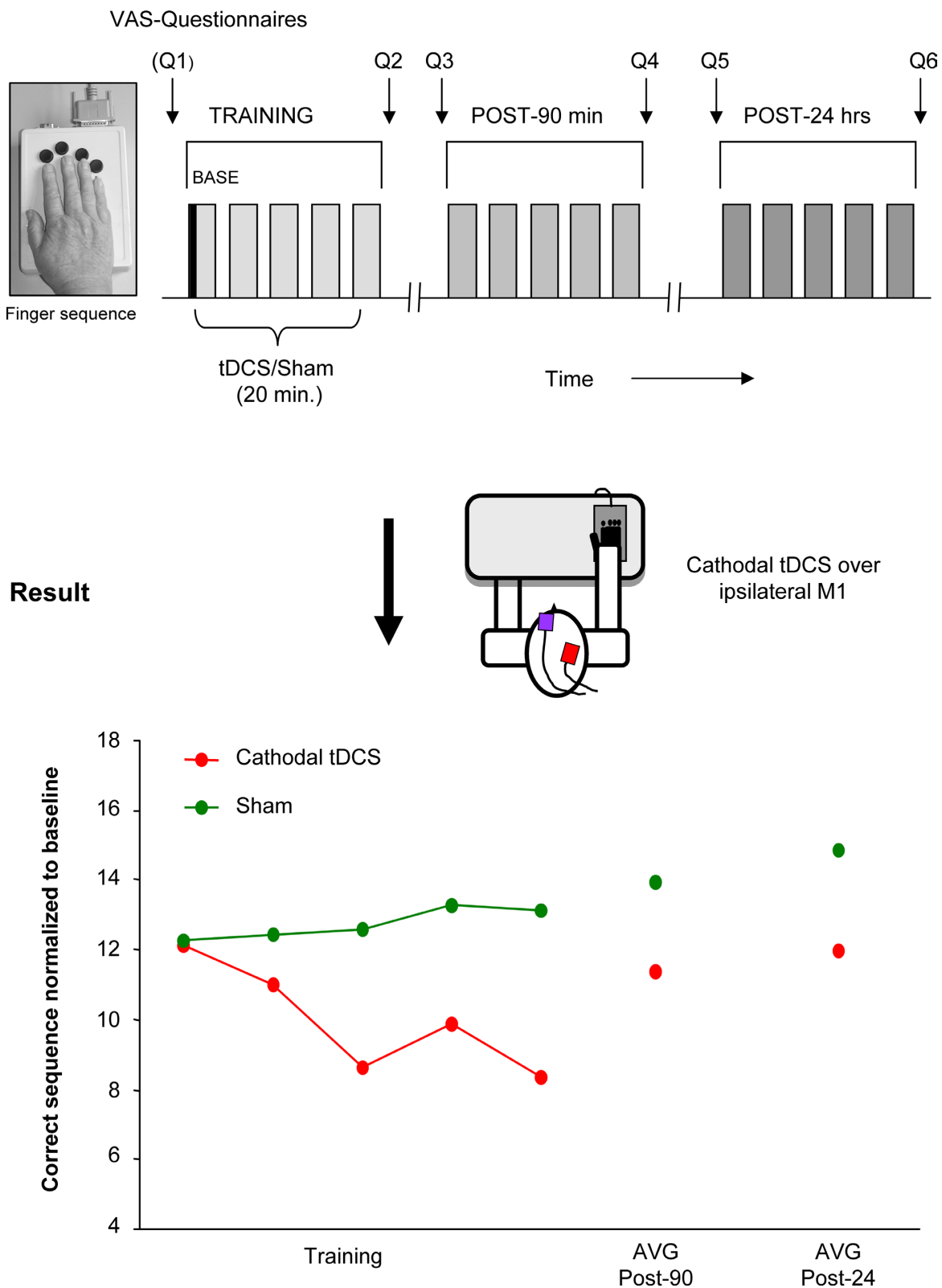

FIGURE 1 | Disruption of function of the ipsilateral motor cortex by tDCS. tDCS disrupting effects on ipsilateral motor cortex during a motor learning task (single subject data). Design: cathodal (inhibitory) tDCS or Sham was applied concurrent with training of a finger sequence, performed with the right hand. The stimulation was delivered in a counterbalanced double-blind design. The motor task was re-evaluated $90 \mathrm{~min}$ and $24 \mathrm{~h}$ after training. Questionnaires $\left(\mathrm{Q}_{1-6}\right)$ regarding attention, fatigue, and hand fatigue were given before and after each session. Results: decreased number of correct sequences was seen with cathodal tDCS applied to the ipsilateral motor cortex but not with Sham stimulation. This data supports the functional relevance of the ipsilateral motor cortex for hand functions and support the view of a compensatory mechanism that takes place in old subjects. (This single subject data is part of a study presented in the international conference of TMS and tDCS by the authors, Gottingen, 2008)
tDCS to simultaneous synaptic activation by low frequency stimulation, induced lasting NMDA receptor dependent synaptic potentiation and this response highly depends on BDNF secretion and TrkB activation (Fritsch et al., 2010).
Given that NIBS techniques and motor learning might share synergistic impacts on synaptic and network plasticity, additive, or even supra-additive effects can be anticipated by applying the innovative combination of NIBS concurrent with training. Along this line, 


\section{Design}

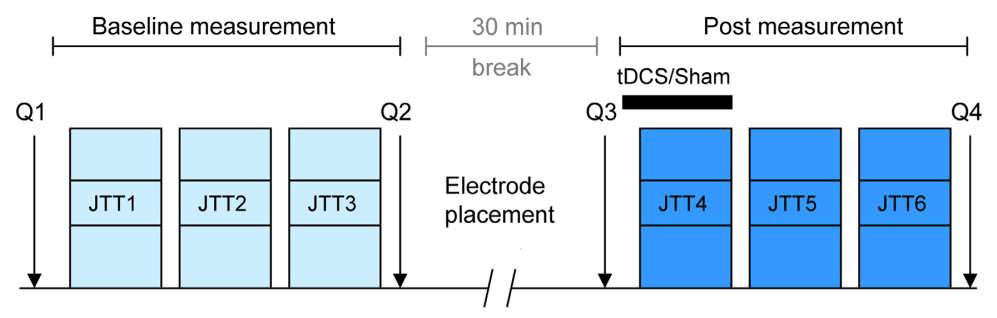

\section{Results}

A

Anodal tDCS

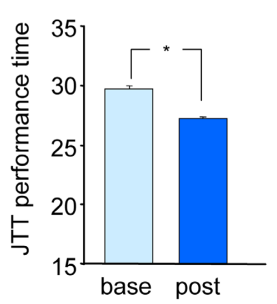

Sham Stimulation

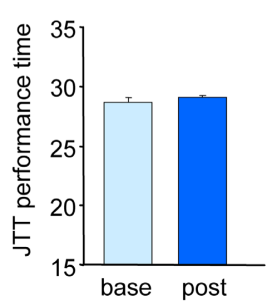

FIGURE 2 | Facilitating skilled motor functions by tDCS. Effect of anodal (excitatory) tDCS applied to the primary motor cortex on the Jebsen-Taylor hand function test (Hummel et al., 2009a). Design: After familiarization with the JTT, subjects took part in two sessions (before and after tDCS) composed of $3 \mathrm{JTT}$. tDCS or Sham was applied during the first 20 min of the second session in a counterbalanced double-blind design. Questionnaires assessing attention and
B

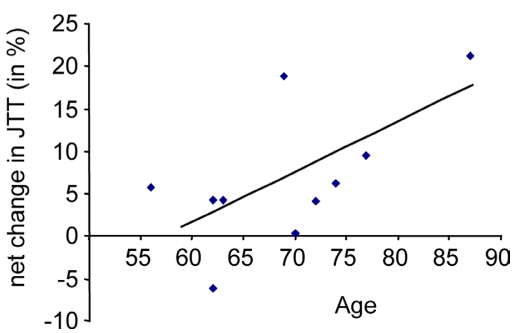

fatigue during the experiment where given $\left(\mathrm{Q}_{1-4}\right)$. Results: (A) tDCS but not Sham stimulation resulted in shorter total time of JTT ( $\mathrm{JTT}_{1-3} \mathrm{VS}_{\mathrm{STT}} \mathrm{JT}_{4-6}$. Performance improvements with tDCS persisted for at least $30 \mathrm{~min}$. (B) Correlation between age and tDCS-induced improvement indicating that the older the subjects the more prominent was the improvement in JTT. (Figure modified from Hummel et al., 2009a).
Zimerman et al. (2010) applied anodal (facilitatory) tDCS to the dominant motor cortex while old healthy subjects trained a complex unimanual finger sequence with the right hand (Zimerman et al., 2010). The study demonstrated a significant increase in skill acquisition by training the movements concurrent with anodal tDCS compared to training with sham intervention, an effect still apparent $24 \mathrm{~h}$ after the offset of tDCS. It is of interest that the slope of the learning curves in elderly increased with the application of tDCS to a level close to demonstrated in young healthy subjects (Figure 3). Taken together, this finding is the first proof-of-principle that combining NIBS with training can dramatically enhance the effects of training in old subjects, who otherwise show impaired motor learning (Brown et al., 2009).

Another strategy probed to increase the effects of NIBS is the repetitive application of NIBS over consecutive days rather than a single session. With this approach, long-lasting effects of up to 3 months have been shown in young healthy subjects (Reis et al., 2009), a promising finding that has to be tested in healthy old subjects.

\section{FUTURE DIRECTIONS}

Based on the above described findings, NIBS techniques are promising tools to study age-related changes in cortical plasticity. They demonstrated the capacity to modulate motor and cognitive functions in this population. There are, however, several open questions regarding underlying mechanisms and the use of NIBS to modulate functions that should be addressed in the near future in upcoming studies before both tDCS and rTMS protocols enter in larger clinical trials.

\section{WHICH PARAMETERS OF rTMS AND tDCS ARE THE MOST EFFECTIVE?}

Based on functional and structure changes that take place in this population and the ambiguity of the previous NIBS results to induce plastic changes in the elder brain (for details please see Table 2). It is conceivable that the protocols that have an effect in the normal young cortex may not have the same effects in an aged cortex. These findings, in deed warrant future studies directed in order to understand more about the basic mechanisms that are involved in neuroplasticity of the aged brain and also of the effects of NIBS on these mechanisms. Moreover, parameters of NIBS might need to be reconsidered in order to induce comparable effects on cortical excitability as in young (e.g., frequency, time, intensity of the NIBS techniques, size of the tDCS electrodes and shape of the TMS coil) and to adjust these parameters to achieve best modulatory effects of NIBS. Therefore, the best parameters for application in old healthy adults have to be further determined (e.g., dose finding). 


\section{Design}
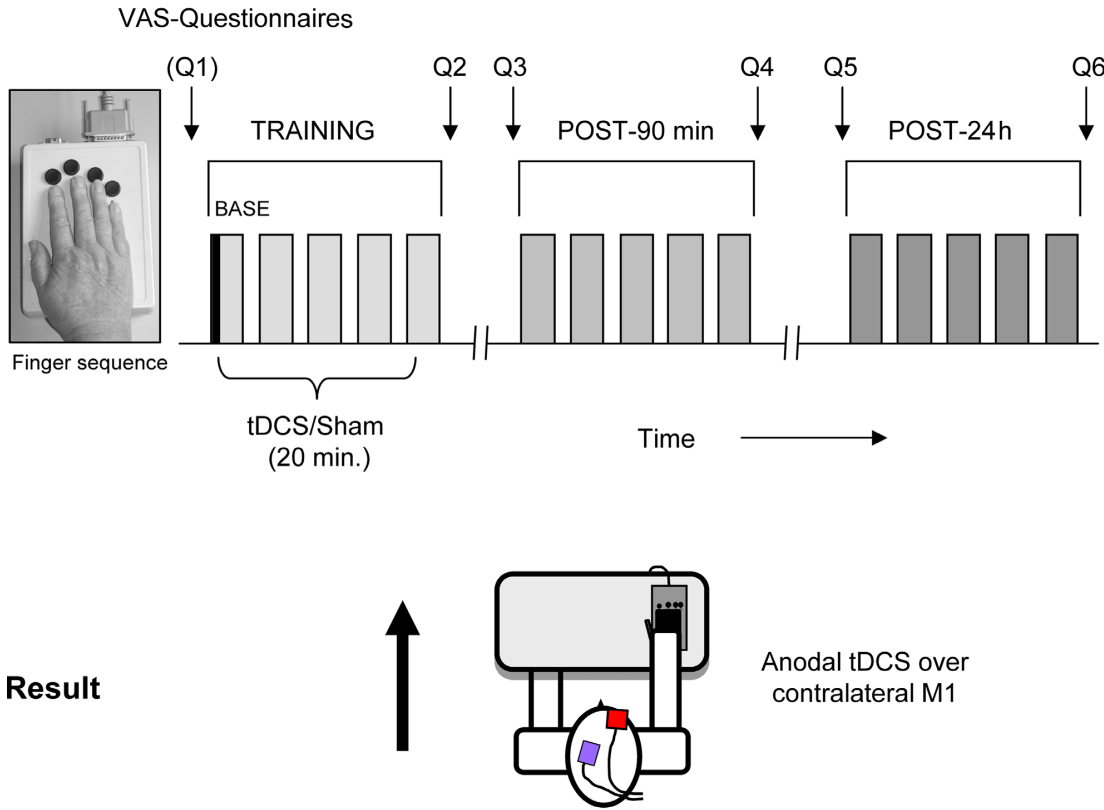

Anodal tDCS over contralateral M1

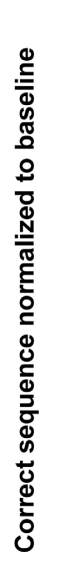

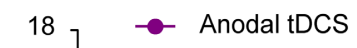

$$
\text { - Sham }
$$

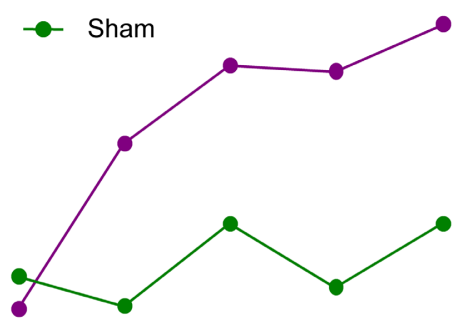

10

6

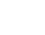

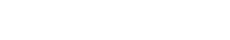




\section{WHICH AREAS ARE THE BEST TARGETS FOR NIBS?}

This is a quite interesting question as recent neuroimaging studies demonstrated large bihemispherical networks involved in the representation of functions in healthy old subjects. Based on this a main question comes to mind, which is not yet fully addressed, and has the potential to provide the basis for sophisticated, neuronavigated NIBS interventions: is a cortical area rather adaptive or maladaptive for a certain function? The primer one would call for NIBS protocols enhancing the activity of this area, in contrast the second one might even call of inhibitory NIBS applied to the respective area. Furthermore, we also need more knowledge about the functional importance of the different cortical areas involved in the presentation of a cognitive function. For example recent neuroimaging study revealed that not all activated cortical areas in the network have the same functional importance during a motor task (Ward et al., 2008). Thus, cortical areas might be more or less promising as a target for NIBS to enhance functions. Until now only anodal tDCS over the contralateral M1 demonstrated to increase skilled motor functions in old subjects (Hummel et al., 2009). The assumption that a widespread and bilateral activation is causally involved in the acquisition and implementation of complex motor acts in old subjects positioned the secondary motor structures as possible target regions for future works. It is tempting to speculate, considering the role of ipsilateral M1, whether increasing the excitability of this area could potentially enhance the performance of a motor task in this population. Probably, the individual network changes induced by aging determine the optimal areas for stimulation. According to this line, future neuroimaging research should be

\section{REFERENCES}

Anderson, B., and Rutledge, V. (1996). Age and hemisphere effects on dendritic structure. Brain 119(Pt 6), 1983-1990.

Antal, A., Nitsche, M. A., Kruse, W., Kincses, T. Z., Hoffmann, K. P., and Paulus, W. (2004). Direct current stimulation over V5 enhances visuomotor coordination by improving motion perception in humans. J. Cogn. Neurosci. 16, 521-527.

Balducci, L., and Extermann, M. (2000). Cancer and aging. An evolving panorama. Hematol. Oncol. Clin. North Am. 14, 1-16.

Bastin, M. E., Maniega, S. M., Ferguson, K. J., Brown, L. J., Wardlaw, J. M., MacLullich, A. M., and Clayden, J. D. (2010). Quantifying the effects of normal ageing on white matter structure using unsupervised tract shape modelling. Neuroimage 51, 1-10.

Bennett, I. J., Madden, D. J., Vaidya, C. J., Howard, D. V., and Howard, J. H. Jr. (2010). Age-related differences in multiple measures of white matter integrity: a diffusion tensor imaging study of healthy aging. Hum. Brain Mapp. 31, 378-390.

Binder, D. K., and Scharfman, H. E. (2004). Brain-derived neurotrophic factor. Growth Factors 22, 123-131.
Boggio, P. S., Campanha, C., Valasek, C. A., Fecteau, S., Pascual-Leone, A., and Fregni, F. (2010). Modulation of decision-making in a gambling task in older adults with transcranial direct current stimulation. Eur. J. Neurosci. 31, 593-597.

Boroojerdi, B., Diefenbach, K., and Ferbert, A. (1996). Transcallosal inhibition in cortical and subcortical cerebral vascular lesions. J. Neurol. Sci. 144, 160-170.

Braver, T. S., and Barch, D. M. (2002). A theory of cognitive control, aging cognition, and neuromodulation. Neurosci. Biobehav. Rev. 26, 809-817.

Brown, R. M., Robertson, E. M., and Press, D.Z. (2009). Sequence skill acquisition and off-line learning in normal aging. PLoS One 4, e6683. doi: 10.1371/journal.pone.0006683.

Buonomano, D. V., and Merzenich, M.M. (1998). Cortical plasticity: from synapses to maps. Annu. Rev. Neurosci. 21, 149-186.

Burke, S. N., and Barnes, C. A. (2006). Neural plasticity in the ageing brain. Nat. Rev. Neurosci. 7, 30-40.

Cabeza, R. (2002). Hemispheric asymmetry reduction in older adults: the HAROLD model. Psychol. Aging 17, 85-100.

Cabeza, R., Anderson, N. D., Locantore, J. K., and McIntosh, A. R. (2002). Aging

performed to establish tailored assessment of age-related changes in cortical activity. Additionally, there is a growing need to extend the possibilities of brain stimulation into other key aspects of aging, e.g., attention, memory, and locomotion.

\section{CONCLUSIONS}

In summary, there is a growing body of evidence suggesting that the aging brain undergoes neuroplastic changes to respond to functional declines and keep performance on the best level. During these changes, additional brain areas are recruited, such as the ipsilateral motor cortex. First proof of principle has been provided that NIBS might modulate cortical functions even in old subjects. Nevertheless, this exciting and progressing field is still at a starting point and more studies are needed to further substantiate the hypothesis that NIBS can be used to enhance functions that have declined with age. In comparison to pharmacological interventions, NIBS is applied focally and does not have systemic side effects, a crucial point to consider in this population. Moreover, these techniques are easy to apply and can be coupled with training protocols or rehabilitative programs, such as physio-, occupational, speech therapy, or gait training to enhance impaired functions with a consecutive improvement of quality of life.

\section{ACKNOWLEDGMENTS}

This research was supported by a grant from the DAAD (German Academic Exchange Service) to Maximo Zimerman (A/07/95990), Alexander von Humboldt Foundation (Feodor-Lynen) to Friedhelm C. Hummel and the Forschungsförderungsfonds Medizin of the University of Hamburg (NWF-04/07 to Friedhelm C. Hummel).

gracefully: compensatory brain activity in high-performing older adults. Neuroimage 17, 1394-1402.

Cabeza, R., McIntosh, A. R., Tulving, E., Nyberg, L., and Grady, C. L. (1997). Age-related differences in effective neural connectivity during encoding and recall. Neuroreport 8, 3479-3483.

Celsis, P. (2000). Age-related cognitive decline, mild cognitive impairment or preclinical Alzheimer's disease? Ann. Med. 32, 6-14.

Cheeran, B., Talelli, P., Mori, F., Koch, G. Suppa, A., Edwards, M., Houlden, H., Bhatia, K., Greenwood, R., and Rothwell, J.C. (2008).A common polymorphism in the brain-derived neurotrophic factor gene (BDNF) modulates human cortical plasticity and the response to rTMS. J. Physiol. 586, 5717-5725.

Chen, R. (2004). Interactions between inhibitory and excitatory circuits in the human motor cortex. Exp. Brain Res. 154, 1-10.

Chen, R., Lozano, A. M., and Ashby, P. (1999). Mechanism of the silent period following transcranial magnetic stimulation. Evidence from epidural recordings. Exp. Brain Res. $128,539-542$.

Cirillo, J., Lavender, A. P., Ridding, M. C., and Semmler, J. G. (2009). Motor cortex plasticity induced by paired associative stimulation is enhanced in physically active individuals. J. Physiol. 587, 5831-5842.

Cohen, L. G., Bandinelli, S., Findley, T. W., and Hallett, M. (1991). Motor reorganization after upper limb amputation in man. A study with focal magnetic stimulation. Brain $114(\mathrm{Pt}$ 1B), 615-627.

Cotelli, M., Manenti, R., Cappa, S. F., Zanetti, O., and Miniussi, C. (2008). Transcranial magnetic stimulation improves naming in Alzheimer disease patients at different stages of cognitive decline. Eur. J. Neurol. 15, 1286-1292.

Craik, F. I., and Bialystok, E. (2006). Planning and task management in older adults: cooking breakfast. Mem. Cognit. 34, 1236-1249.

Duque, J., Mazzocchio, R., Dambrosia, J., Murase, N., Olivier, E., and Cohen, L. G. (2005). Kinematically specific interhemispheric inhibition operating in the process of generation of a voluntary movement. Cereb. Cortex 15, 588-593.

Egan, M. F., Kojima, M., Callicott, J. H., Goldberg, T. E., Kolachana, B. S., Bertolino, A., Zaitsev, E., Gold, B., Goldman, D., Dean, M., Lu, B., and Weinberger, D. R. (2003). The BDNF 
val66met polymorphism affects activity-dependent secretion of BDNF and human memory and hippocampal function. Cell 112, 257-269.

Eisen,A., Entezari-Taher,M., and Stewart, H. (1996). Cortical projections to spinal motoneurons: changes with aging and amyotrophic lateral sclerosis. Neurology 46, 1396-1404.

Fathi, D., Ueki, Y., Mima, T., Koganemaru, S., Nagamine, T., Tawfik, A., and Fukuyama, H. (2009). Effects of aging on the human motor cortical plasticity studied by paired associative stimulation. Clin. Neurophysiol. 121, 90-93.

Ferbert, A., Priori, A., Rothwell, J. C., Day, B. L., Colebatch, J. G., and Marsden, C. D. (1992). Interhemispheric inhibition of the human motor cortex. J. Physiol. $453,525-546$.

Ferrucci, R., Mameli, F., Guidi, I., MrakicSposta, S., Vergari, M., Marceglia, S., Cogiamanian, F., Barbieri, S., Scarpini, E., and Priori, A. (2008). Transcranial direct current stimulation improves recognition memory in Alzheimer disease. Neurology 71, 493-498.

Floel,A., Vomhof, P., Lorenzen, A., Roesser, N., Breitenstein, C., and Knecht, S. (2008). Levodopa improves skilled hand functions in the elderly. Eur. J. Neurosci. 27, 1301-1307.

Fregni, F., and Pascual-Leone, A. (2007). Technology insight: noninvasive brain stimulation in neurology-perspectives on the therapeutic potential of rTMS and tDCS. Nat. Clin. Pract. Neurol. 3 , 383-393.

Fritsch, B., Reis, J., Martinowich, K., Schambra, H. M., Ji, Y., Cohen, L. G., and Lu, B. (2010). Direct current stimulation promotes BDNF-dependent synaptic plasticity: potential implications for motor learning. Neuron 66, 198-204.

Fujiki, M., and Steward, O. (1997). High frequency transcranial magnetic stimulation mimics the effects of ECS in upregulating astroglial gene expression in the murine CNS. Brain Res. Mol. Brain Res. 44, 301-308.

Gandiga, P.C., Hummel, F.C., and Cohen, L. G. (2006). Transcranial DC stimulation (tDCS): a tool for double-blind sham-controlled clinical studies in brain stimulation. Clin. Neurophysiol. $117,845-850$.

Hallett, M. (1995). Transcranial magnetic stimulation. Negative effects. $A d v$. Neurol. 67, 107-113.

Hallett, M. (2007). Transcranial magnetic stimulation: a primer. Neuron $55,187-199$.

Hanajima, R., Ugawa, Y., Terao, Y., Sakai, K., Furubayashi, T., Machii, K., and Kanazawa, I. (1998). Paired-pulse magnetic stimulation of the human motor cortex: differences among I waves. J. Physiol. 509(Pt 2), 607-618.
Hausmann, A., Weis, C., Marksteiner, J., Hinterhuber, H., and Humpel, C. (2000). Chronic repetitive transcranial magnetic stimulation enhances c-fos in the parietal cortex and hippocampus. Brain Res. Mol. Brain Res. 76, 355-362.

Heise, K. F., Steven, B., Liuzzi, G., Thomalla, G.,Jonas,M.,Muller-Vahl,K.,Sauseng,P., Munchau, A., Gerloff, C., and Hummel, F. C. (2010). Altered modulation of intracortical excitability during movement preparation in Gilles de la Tourette syndrome. Brain 133, 580-590.

Herrmann, M. J., Walter, A., Ehlis, A. C., and Fallgatter, A. J. (2006). Cerebral oxygenation changes in the prefrontal cortex: effects of age and gender. Neurobiol. Aging 27, 888-894.

Hogan, M. J., Kelly, C. A., and Craik, F. I. (2006). The effects of attention switching on encoding and retrieval of words in younger and older adults. Exp. Aging Res. 32, 153-183.

Huang, Y. Z., Edwards, M. J., Rounis, E., Bhatia, K. P., and Rothwell, J.C. (2005). Theta burst stimulation of the human motor cortex. Neuron 45, 201-206.

Hummel, F. C., and Cohen, L. G. (2005). Drivers of brain plasticity. Curr. Opin. Neurol. 18, 667-674.

Hummel, F., Voller, B., Celnik, P., Floel, A., Giraux, P., Gerloff, C., and Cohen, L. G. (2006). Effects of brain polarization on reaction times and pinch force in chronic stroke. BMC Neurosci. 7, 73. doi: 10.1186/1471-2202-7-73.

Hummel, F. C., and Cohen, L. G. (2006). Non-invasive brain stimulation: a new strategy to improve neurorehabilitation after stroke? Lancet Neurol. 5, 708-712.

Hummel, F. C., Celnik, P., Giraux, P., Floel, A., Wu, W. H., Gerloff, C., and Cohen, L. G. (2005). Effects of non-invasive cortical stimulation on skilled motor function in chronic stroke. Brain 128, 490-499.

Hummel, F. C., Heise, K., Celnik, P., Floel, A., Gerloff, C., and Cohen, L. G. (2009a). Facilitating skilled right hand motor function in older subjects by anodal polarization over the left primary motor cortex. Neurobiol. Aging. 31, 2160-2168.

Hummel, F. C., Steven, B., Hoppe, J., Heise, K., Thomalla, G., Cohen, L. G., and Gerloff, C. (2009b). Deficient intracortical inhibition (SICI) during movement preparation after chronic stroke. Neurology 72, 1766-1772.

Huntley, G. W. (1997). Correlation between patterns of horizontal connectivity and the extend of short-term representational plasticity in rat motor cortex [see comments]. Cereb. Cortex 7, 143-156.

Jenkins, W. M., Merzenich, M. M., and Recanzone, G. (1990). Neocortical representational dynamics in adult primates: implications for neuropsychology. Neuropsychologia 28, 573-584.

Ji, R. R., Schlaepfer, T.E., Aizenman, C. D., Epstein, C. M., Qiu, D., Huang, J. C., and Rupp, F. (1998). Repetitive transcranial magnetic stimulation activates specific regions in rat brain. Proc. Natl. Acad. Sci. U.S.A. 95, 15635-15640.

Jones, E. G. (1993). GABAergic neurons and their role in cortical plasticity in primates. Cereb. Cortex 3, 361-372.

Kaelin-Lang, A., Luft, A. R., Sawaki, L., Burstein, A. H., Sohn, Y.H., and Cohen, L.G. (2002). Modulation of human corticomotor excitability by somatosensory input. J. Physiol. 540, 623-633.

Kleim, J.A., Chan, S., Pringle, E., Schallert, K., Procaccio, V., Jimenez, R., and Cramer, S. C. (2006). BDNF val66met polymorphism is associated with modified experience-dependent plasticity in human motor cortex. Nat. Neurosci. 9, 735-737.

Komiyama, T., Sato, T. R., O'Connor, D. H., Zhang, Y. X., Huber, D., Hooks, B. M., Gabitto, M., and Svoboda, K. (2010) Learning-related fine-scale specificity imaged in motor cortex circuits of behaving mice. Nature 464, 1182-1186.

Krampe, R. T., Engbert, R., and Kliegl, R. (2002). The effects of expertise and age on rhythm production: adaptations to timing and sequencing constraints. Brain Cogn. 48, 179-194.

Kujirai, T., Caramia, M.D., Rothwell, J.C., Day, B. L., Thompson, P. D., Ferbert, A. Wroe, S., Asselman, P., and Marsden, C. D. (1993). Corticocortical inhibition in human motor cortex. J. Physiol. (Lond.) 471, 501-519.

Logsdon, R. G., Gibbons, L. E., McCurry, S. M., and Teri, L. (2002). Assessing quality of life in older adults with cognitive impairment. Psychosom. Med. 64, 510-519.

Mattay, V. S., Fera, F., Tessitore, A., Hariri, A. R., Das, S., Callicott, J. H., and Weinberger, D. R. (2002) Neurophysiological correlates of agerelated changes in human motor function. Neurology 58, 630-635.

McGinley, M., Hoffman, R. L., Russ, D.W. Thomas, J. S., and Clark, B. C. (2010). Older adults exhibit more intracorti$\mathrm{cal}$ inhibition and less intracortical facilitation than young adults. Exp. Gerontol. 45, 671-678.

Muller,M.B., Toschi,N., Kresse,A.E.,Post,A., and Keck,M.E. (2000).Long-term repetitive transcranial magnetic stimulation increases the expression of brain-derived neurotrophic factor and cholecystokinin mRNA, but not neuropeptide tyrosine mRNA in specific areas of rat brain. Neuropsychopharmacology 23 , 205-215.

Muller-Dahlhaus, J. F., Orekhov, Y., Liu, Y., and Ziemann, U. (2008).
Interindividual variability and age-dependency of motor cortical plasticity induced by paired associative stimulation. Exp. Brain Res. 187, 467-475.

Nielson, K. A., Langenecker, S. A., and Garavan, H. (2002). Differences in the functional neuroanatomy of inhibitory control across the adult life span. Psychol. Aging 17, 56-71.

Nitsche, M.A., Cohen, L., Wassermann, E. Priori,A., Lang, N., Antal,A., Paulus, W., Hummel, F., Boggio, S., Fregni, F., and Pascual-Leone, A. (2008). Transcranial direct current stimulation: state of the art 2008. Brain Stimul. 1, 206-223.

Nitsche, M. A., Fricke, K., Henschke, U., Schlitterlau, A., Liebetanz, D., Lang, N., Henning, S., Tergau, F., and Paulus, W. (2003a). Pharmacological modulation of cortical excitability shifts induced by transcranial direct current stimulation in humans. J. Physiol. 553, 293-301.

Nitsche,M.A.,Liebetanz,D.,Antal,A.,Lang, N., Tergau, F., and Paulus, W. (2003b). Modulation of cortical excitability by weak direct current stimulation technical, safety and functional aspects. Suppl. Clin. Neurophysiol. 56, 255-276.

Nitsche,M.A.,Jaussi,W.,Liebetanz,D.,Lang, N., Tergau, F., and Paulus, W. (2004). Consolidation of human motor cortical neuroplasticity by $\mathrm{D}$-cycloserine. Neuropsychopharmacology 29, 1573-1578.

Nitsche, M. A., Lampe, C., Antal, A., Liebetanz, D., Lang, N., Tergau, F., and Paulus, W. (2006). Dopaminergic modulation of long-lasting direct current-induced cortical excitability changes in the human motor cortex, 73. Eur. J. Neurosci. 23, 1651-1657.

Oliviero, A., Profice, P., Tonali, P. A., Pilato, F., Saturno, E., Dileone, M., Ranieri, F., and Di Lazzaro, V. (2006). Effects of aging on motor cortex excitability. Neurosci. Res. 55, 74-77.

Peinemann, A., Lehner, C., Conrad, B., and Siebner, H. R. (2001). Age-related decrease in paired-pulse intracortical inhibition in the human primary motor cortex. Neurosci. Lett. 313, 33-36.

Pellicciari, M. C., Miniussi, C., Rossini, P. M., and De Gennaro, L. (2009). Increased cortical plasticity in the elderly: changes in the somatosensory cortex after paired associative stimulation. Neuroscience 163, 266-276.

Persson, J., Nyberg, L., Lind, J., Larsson, A., Nilsson, L. G., Ingvar, M., and Buckner, R. L. (2006). Structure-function correlates of cognitive decline in aging. Cereb. Cortex 16, 907-915.

Pierpaoli, C., Jezzard,P., Basser,P.J., Barnett, A., and Di Chiro, G. (1996). Diffusion tensor MR imaging of the human brain. Radiology 201, 637-648.

Quartarone, A., Lang, N., Rizzo, V., Bagnato, S., Morgante, F., Sant'angelo, 
A., Crupi, D., Battaglia, F., Messina, C., and Girlanda, P. (2007). Motor cortex abnormalities in amyotrophic lateral sclerosis with transcranial directcurrent stimulation. Muscle Nerve 35, 620-624.

Reis, J., Schambra, H. M., Cohen, L. G., Buch, E. R., Fritsch, B., Zarahn, E., Celnik, P. A., and Krakauer, J. W. (2009). Noninvasive cortical stimulation enhances motor skill acquisition over multiple days through an effect on consolidation. Proc. Natl. Acad. Sci. U.S.A. 106, 1590-1595.

Resnick, S. M., Pham, D. L., Kraut, M. A., Zonderman, A. B., and Davatzikos, C. (2003). Longitudinal magnetic resonance imaging studies of older adults: a shrinking brain. J. Neurosci. 23, 3295-3301.

Reuter-Lorenz, P. A., Jonides, J., Smith, E. E., Hartley, A., Miller, A., Marshuetz, C., and Koeppe, R. A. (2000). Age differences in the frontal lateralization of verbal and spatial working memory revealed by PET. J. Cogn. Neurosci. 12, 174-187.

Reynolds, C., and Ashby, P. (1999). Inhibition in the human motor cortex is reduced just before a voluntary contraction. Neurology 53, 730-735.

Ridding, M. C., and Ziemann, U. (2010). Determinants of the induction of cortical plasticity by non-invasive brain stimulation in healthy subjects. J. Physiol. 588, 2291-2304.

Rioult-Pedotti, M. S., Friedman, D., and Donoghue, J. P. (2000). Learninginduced LTP in neocortex. Science 290, 533-536.

Rogasch, N. C., Dartnall, T. J., Cirillo, J., Nordstrom, M. A., and Semmler, J. G. (2009). Corticomotor plasticity and learning of a ballistic thumb training task are diminished in older adults. J. Appl. Physiol. 107, 1874-1883.

Rosenkranz, K., Kacar, A., and Rothwell, J. C. (2007). Differential modulation of motor cortical plasticity and excitability in early and late phases of human motor learning. J. Neurosci.27, 12058-12066.

Rossi, S., Hallett, M., Rossini, P. M., and Pascual-Leone, A. (2009). Safety, ethical considerations, and application guidelines for the use of transcranial magnetic stimulation in clinical practice and research. Clin. Neurophysiol. 120, 2008-2039.

Roth, G. S., and Joseph, J. A. (1994). Cellular and molecular mechanisms of impaired dopaminergic function during aging. Ann. N. Y. Acad. Sci. 719, 129-135.

Sailer, A., Dichgans, J., and Gerloff, C. (2000). The influence of normal aging on the cortical processing of a simple motor task. Neurology 55, 979-985.
Sale,M.V., Ridding, M.C., and Nordstrom, M. A. (2007). Factors influencing the magnitude and reproducibility of corticomotor excitability changes induced by paired associative stimulation. Exp. Brain Res. 181, 615-626.

Sale, M.V., and Semmler, J. G. (2005). Agerelated differences in corticospinal control during functional isometric contractions in left and right hands. J. Appl. Physiol. 99, 1483-1493.

Sawaki, L., Yaseen, Z., Kopylev, L., and Cohen, L. G. (2003). Age-dependent changes in the ability to encode a novel elementary motor memory. Ann. Neurol. 53, 521-524.

Scherder, E., Dekker, W., and Eggermont, L. (2008). Higher-level hand motor function in aging and (preclinical) dementia: its relationship with (instrumental) activities of daily life - a minireview. Gerontology 54, 333-341.

Schmidt, S., Redecker, C., Bruehl, C., and Witte, O. W. (2010). Age-related decline of functional inhibition in rat cortex. Neurobiol. Aging 31, 504-511.

Siebner, H. R., Lang, N., Rizzo, V., Nitsche, M. A., Paulus, W., Lemon, R. N., and Rothwell,J.C. (2004). Preconditioning of low-frequency repetitive transcranial magnetic stimulation with transcranial direct current stimulation: evidence for homeostatic plasticity in the human motor cortex. J. Neurosci. 24, 3379-3385.

Silvanto, J., Muggleton, N., and Walsh, V. (2008). State-dependency in brain stimulation studies of perception and cognition. Trends Cogn. Sci. 12, 447-454.

Stadlbauer, A., Salomonowitz, E., Strunk, G., Hammen, T., and Ganslandt, O. (2008). Age-related degradation in the central nervous system: assessment with diffusion-tensor imaging and quantitative fiber tracking. Radiology 247, 179-188.

Stebbins, G. T., Carrillo, M. C., Dorfman, J., Dirksen, C., Desmond, J. E., Turner D. A., Bennett, D. A., Wilson, R. S., Glover, G., and Gabrieli, J. D. (2002). Aging effects on memory encoding in the frontal lobes. Psychol. Aging $17,44-55$.

Stefan, K., Kunesch, E., Cohen, L. G., Benecke, R., and Classen, J. (2000). Induction of plasticity in the human motor cortex by paired associative stimulation. Brain $123(\mathrm{Pt} \mathrm{3})$, 572-584.

Strafella, A. P., Paus, T., Fraraccio, M., and Dagher, A. (2003). Striatal dopamine release induced by repetitive transcranial magnetic stimulation of the human motor cortex. Brain 126, 2609-2615.

Sullivan, E.V., and Pfefferbaum,A. (2006) Diffusion tensor imaging and aging. Neurosci. Biobehav. Rev. 30, 749-761.
Talelli, P., Ewas, A., Waddingham, W., Rothwell, J.C., and Ward, N. S. (2008a). Neural correlates of age-related changes in cortical neurophysiology. Neuroimage 40, 1772-1781.

Talelli, P., Waddingham, W., Ewas, A. Rothwell, J. C., and Ward, N. S. (2008b). The effect of age on task-related modulation of interhemispheric balance. Exp. Brain Res. 186, 59-66.

Tecchio, F., Zappasodi, F., Pasqualetti, P., De Gennaro, L., Pellicciari, M. C. Ercolani, M., Squitti, R., and Rossini, P. M. (2008). Age dependence of primary motor cortex plasticity induced by paired associative stimulation. Clin. Neurophysiol. 119, 675-682.

Todd, G., Kimber, T. E., Ridding, M. C. and Semmler, J. G. (2010). Reduced motor cortex plasticity following inhibitory rTMS in older adults. Clin. Neurophysiol. 121, 441-447.

Toescu, E.C., Verkhratsky,A., and Landfield, P.W. (2004). Ca2+ regulation and gene expression in normal brain aging. Trends Neurosci. 27, 614-620.

UN-Report. (2005). Human Development Report 2005. International cooperation at a crossroads-aid, trade and security in an unequal world. United Nations ISBN 97.90195305111, New York.

Volkow, N. D., Wang, G. J., Fowler, J. S. Ding, Y.S., Gur, R. C., Gatley, J., Logan, J., Moberg, P. J., Hitzemann, R., Smith, G., and Pappas, N. (1998). Parallel loss of presynaptic and postsynaptic dopamine markers in normal aging. Ann. Neurol. 44, 143-147.

Ward, N. S., and Frackowiak, R. S. (2003) Age-related changes in the neural correlates of motor performance. Brain $126,873-888$.

Ward, N. S., Swayne, O. B., and Newton, J M. (2008). Age-dependent changes in the neural correlates of force modulation: an fMRI study. Neurobiol. Aging 29, 1434-1446.

Wassermann, E. M. (1998). Risk and safety of repetitive transcranial magnetic stimulation: report and suggested guidelines from the International Workshop on the Safety of Repetitive Transcranial Magnetic Stimulation, June 5-7, 1996. Electroencephalogr. Clin. Neurophysiol. 108, 1-16.

Wassermann, E. M. (2002). Variation in the response to transcranial magnetic brain stimulation in the general population. Clin. Neurophysiol. 113 1165-1171.

Werhahn, K. J., Kunesch, E., Noachtar, S. Benecke, R., and Classen, J. (1999). Differential effects on motorcortical inhibition induced by blockade of GABA uptake in humans. J. Physiol. 517(Pt 2), 591-597.

Wu, A. D., Fregni, F., Simon, D. K. Deblieck, C., and Pascual-Leone, A (2008). Noninvasive brain stimu- lation for Parkinson's disease and dystonia. Neurotherapeutics 5 , 345-361.

Yu, Z. Y., Wang, W., Fritschy, J. M., Witte, O. W., and Redecker, C. (2006). Changes in neocortical and hippocampal GABAA receptor subunit distribution during brain maturation and aging. Brain Res. 1099, 73-81.

Zaman, V., Boger, H. A., Granholm, A. C., Rohrer, B., Moore, A., Buhusi, M., Gerhardt, G. A., Hoffer, B. J., and Middaugh, L. D. (2008). The nigrostriatal dopamine system of aging GFRalpha-1 heterozygous mice: neurochemistry, morphology and behavior. Eur. J. Neurosci. 28, 1557-1568.

Ziemann, U., Lonnecker, S., Steinhoff, B. J., and Paulus, W. (1996). Effects of antiepileptic drugs on motor cortex excitability in humans: a transcranial magnetic stimulation study. Ann. Neurol. 40, 367-378.

Ziemann, U., Tam, A., Butefisch, C., and Cohen, L. G. (2002). Dual modulating effects of amphetamine on neuronal excitability and stimulation-induced plasticity in human motor cortex. Clin. Neurophysiol. 113, 1308-1315.

Zimerman, M., Nitsch, M., Cohen, L. G., Gerloff, C., and Hummel, F. (2008). "The functional role of the ipsilateral motor cortex for motor sequence learning in the elderly," in Proceedings of the Third International Conference on Transcranial Magnetic and Direct Current Stimulation, Gottingen 2008.

Zimerman, M., Nitsch, M., Giraux, P. Liuzzi, G., Cohen, L. G., Gerloff, C., and Hummel, F. C. (2010). "Neuroenhancement of the aging brain: restoring skill acquisition in the elderly," in Proceedings of the Sixth World Congress for Neurorehabilitation, Vienna 2010 (Poster session).

Conflict of Interest Statement: The authors declare that the research was conducted in the absence of any commercial or financial relationships that could be construed as a potential conflict of interest

Received: 11 June 2010; accepted: 20 October 2010; published online: 01 December 2010.

Citation: Zimerman $M$ and Hummel FC (2010) Non-invasive brain stimulation: enhancing motor and cognitive functions in healthy old subjects. Front. Ag. Neurosci. 2:149. doi: 10.3389/fnagi.2010.00149

Copyright $(2010$ Zimerman and Hummel. This is an open-access article subject to an exclusive license agreement between the authors and the Frontiers Research Foundation, which permits unrestricted use, distribution, and reproduction in any medium, provided the original authors and source are credited. 